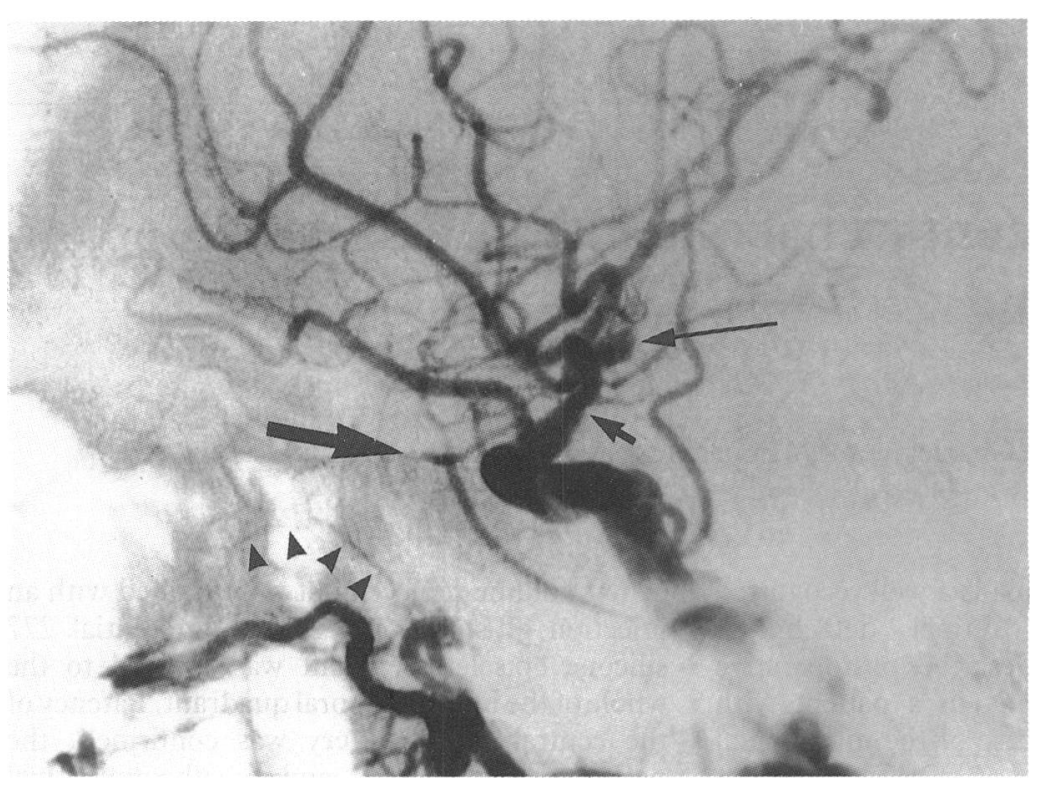

Figure 2 Left common carotid angiogram demonstrating left middle cerebral artery aneurysm (long arrow). There is a quite severe atheromatous stricture of the post cavernous internal carotid (short arrow). The ophthalmic artery is occluded immediately distal to the optic canal (large arrow) and the central retinal artery is fed by branches of the maxillary division of the external carotid artery (arrowheads).

has been attributed to several factors, including interruption of the anterior ciliary arteries, damage to the long posterior ciliary arteries, and compression of vortex veins reducing uveal blood flow. ${ }^{2}$ In our case there was no encircling element placed around the eye and no interference with the anterior or posterior ciliary vessels. Anterior segment ischaemia has only rarely been described following use of a segmental buckle. ${ }^{3}$

Chronic anterior segment ischaemia as part of the panocular ischaemia seen with carotid occlusive disease has been described. ${ }^{4}$ In the case we report it is suggested that the atheromatous disease of the internal carotid artery demonstrated previously, with presumably an associated reduction in ophthalmic artery flow, was of significance in the precipitation of acute anterior segment ischaemia following routine nonencircling retinal detachment surgery. The sub- arachnoid haemorrhage and need for aneurysm clipping is not thought to be of significance in the precipitation of anterior segment ischaemia but has provided angiographic evidence of significant co-existing atheromatous disease.

Collateral circulations in the anterior segment have been described, including superficial marginal plexus at the limbus, episcleral limbal plexus, the intramuscular circulation in the ciliary body, and the major arteriolar circle in the iris root. Experimental evidence in animals has been presented to show that a $360^{\circ}$ peritomy has a demonstrable effect in the production of anterior segment ischaemia following strabismus surgery. ${ }^{5}$ Our patient may have had a precarious ocular blood supply before detachment surgery further compromised by the $360^{\circ}$ conjunctival peritomy sufficient to interrupt the blood supply to his anterior segment.

It has been shown that the blood flow rates in the major temporal retinal arteries following uncomplicated scleral buckling is on average $50 \%$ lower than in the unoperated contralateral eye. ${ }^{6}$ Removal of the scleral buckling element has been shown to increase arterial blood flow rates. In the presence of suspected predisposing factors such as carotid occlusive disease appropriate measures may be taken to avoid anterior segment ischaemia. These include a smaller and more posterior conjunctival incision and adopting an appropriate vitreoretinal approach that avoids extensive buckling and encircling procedures.

l Ryan SJ, Goldberg MF. Anterior segment ischemia following scleral buckling in sickle cell hemoglobinopathy. Am $\mathcal{F}$ Ophthalmol 1971; 72: 35-50.

2 Hayreh SS, Baines JAB. Occlusion of the vortex veins. Br f Ophthalmol 1973; 57: 217-38.

3 Robertson DM. Anterior segment ischemia after segmental episcleral buckling and cryopexy. Am $\mathcal{F}$ Ophthalmol 1975; 79: $871-4$.

4 Dugan JD, Green WR. Ophthalmic manifestations of carotid occlusive disease. Eye 1991; 5: 226-38.

5 Fishman PH, Repta MX, Green WR, D'Anna SA, Guyton DL. A primate model of anterior segment ischemia after A primate model of anterior segment ischemia

6 Ogasawara H, Feke GT, Yoshida A, Milbocker MT, Weiter JJ McMeel JW. Retinal blood flow alterations associated with scleral buckling and encircling procedures. Br $\mathcal{F}$ Ophthalmol 1992; 76: 275-9.
Department of Ophthalmology, University of Leicester, Leicester Royal Infirmary

J M Sparrow

A R Rosenthal

Correspondence to: Mr N A Frost, Department of Ophthalmology, Leicester Royal Infirmary, Leicester LE1 5WW.

Accepted for publication 13 December 1993

\title{
Posterior scleritis with retinal vasculitis and choroidal and retinal infarction
}

\author{
N Andrew Frost, John M Sparrow, A Ralph Rosenthal
}

Posterior scleritis is a rare but probably underdiagnosed disease process. The clinical features are variable and the lesions may cause diagnostic confusion. Visual impairment is common but visual loss due directly to vascular involvement is unusual. In the case presented vascular involvement was exceptionally severe and determined the long term visual outcome. 


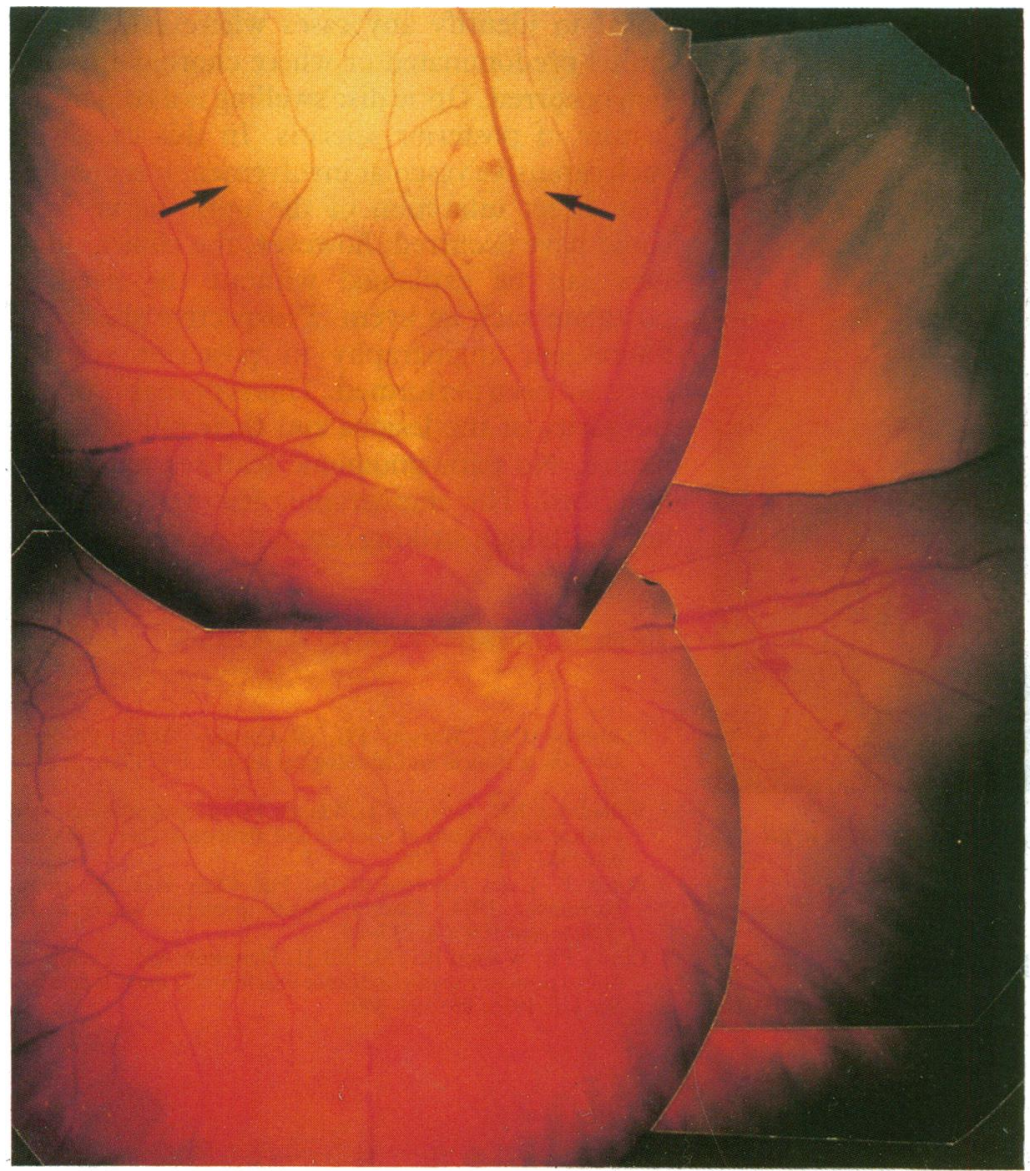

Figure 1 Posterior scleritis: retinal oedema, haemorrhages, swollen optic disc, and vascular stasis. Note outer retinal oedema overlying segmental choroidal infarction (between arrows).

\section{Case report}

A 34-year-old white woman presented with a 3 day history of constant pain in the right eye and right temporal region followed by visual deterioration. She had no symptoms of systemic illness and had no significant medical or family history. On examination there was uncertain perception of light in the right eye, with a right afferent pupillary defect. The right anterior sclera was obscured by gross conjunctival chemosis. A

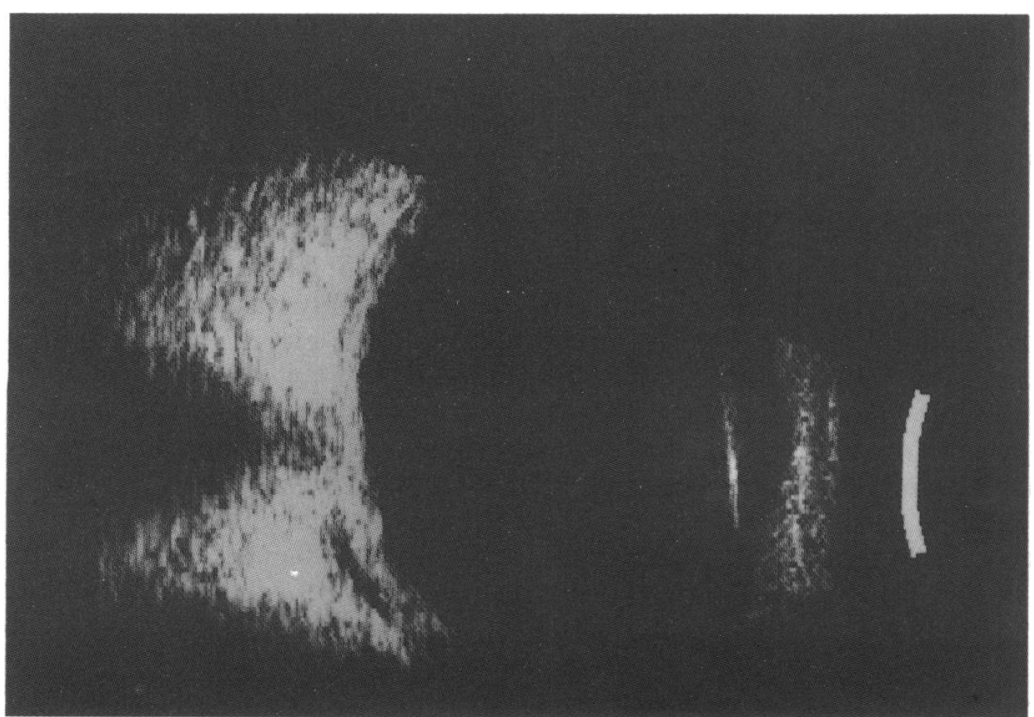

Figure 2 Posterior scleritis: scleral thickening at the posterior pole and retrobulbar oedema. generalised restriction of eye movement was found together with mild proptosis $(2 \mathrm{~mm})$. Mild anterior uveitis was present. There was no vitreous haze. Intraocular pressures were $8 \mathrm{~mm} \mathrm{Hg}$ (right) and $14 \mathrm{~mm} \mathrm{Hg}$ (left). The right retina was widely oedematous and ischaemic with intraretinal haemorrhages, segmentation of the blood column within retinal vessels, and swollen optic disc (Fig 1). The left eye was normal. General physical examination was normal apart from mild pyrexia $\left(37 \cdot 4^{\circ} \mathrm{C}\right)$.

Investigations were performed as follows: full blood count, urea and electrolytes, calcium, liver enzymes, and urate were normal; plasma viscosity $1.77 \mathrm{cp}$; C-reactive protein $1.1 \mathrm{mg} / \mathrm{dl}$; C3, C4 normal; ANA (hep-2) negative; ANCA negative; A-ENA negative; VDRL and TPHA negative; creatinine clearance normal; chest $x$ ray normal. B mode ultrasound scan of the right eye and orbit showed scleral thickening at the posterior pole and retrobulbar oedema (Fig 2). The retrobulbar optic nerve shadow appeared normal. There was no evidence of other orbital pathology. Fluorescein angiography demonstrated retinal vasculitis with multiple branch retinal arterial occlusions and vessel staining together with segmental choroidal ischaemia (Fig 3).

A diagnosis of posterior scleritis was made and she was treated with high dose oral prednisolone, together with topical corticosteroids and atropine. The inflammation responded rapidly but the retina was extensively infarcted and the choroid was segmentally infarcted in several areas. The visual acuity remaining was 'hand movements' only. The systemic corticosteroid therapy was uneventfully reduced over approximately 5 months. At 16 months' follow up there was no evidence of recurrent local inflammation or systemic disease. The anterior sclera appeared normal but funduscopy revealed gross right optic atrophy with wedge shaped areas of choroidoretinal atrophy and many attenuated and nonperfused retinal vessels. Her visual acuity remained unchanged.

\section{Comment}

In this case the diagnosis of posterior scleritis was made clinically and supported by the ultrasonographic findings. The diagnosis has been further supported by the rapid response to corticosteroid therapy and the absence of other systemic or local orbital disease over 16 months of follow up. Posterior scleritis is frequently accompanied by systemic vasculitis but, surprisingly, visual impairment directly caused by vascular involvement is poorly documented. Four cases of choroidal vasculitis (without choroidal infarction), four cases with retinal vascular cuffing (without retinal vasculitis), and one case with subclinical retinal vasculitis, detected by fluorescein angiography were documented by Calthorpe et al. ${ }^{1}$ Juxtapapillary scleral inflammation suggesting direct extension to the central retinal vessels has been described. ${ }^{2} \mathrm{~A}$ case of posterior scleritis with bullous exudative retinal detachment followed by 'segmentary retinal phlebitis' at the posterior pole was described by Bonin. ${ }^{3}$ Intraretinal haemorrhages in the area of a 


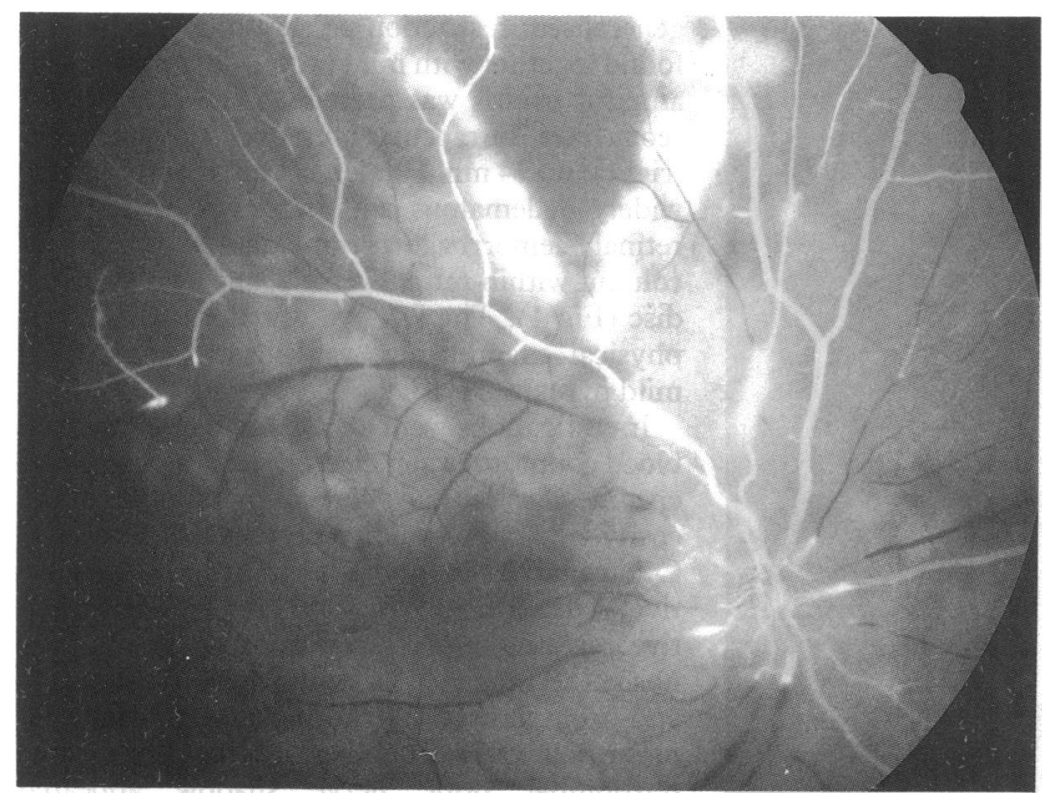

Figure 3 Posterior scleritis: fluorescein angiogram demonstrating multiple retinal arterial occlusions with vessel staining and segmental choroidal ischaemia.

retinal detachment were also found in one case by Cleary et $a l .{ }^{4}$ Review of the current literature failed to identify any cases where retinal vasculitis predominated or where choroidal infarction occurred. Optic disc swelling is a recognised feature of posterior scleritis. In this case with such extensive vascular involvement it is possible that direct infarction of the optic nerve head could have occurred; however, the clinical findings can be explained without the need to postulate such an event. Prospective studies of fluorescein angiography in posterior scleritis have not been performed. This is probably due to the rarity of the lesions and their polymorphic nature. No pathognomonic features have been described. The angiographic features described here, although striking, are not specific.

The photographs were taken by Mr Hugh Harris.

1 Calthorpe CM, Watson PG, McCartney ACE. Posterior scleritis: a clinical and histological survey. Eye 1988; 2: 267scle.

2 Wilhelmus KR, Grierson I, Watson PG. Histopathologic and clinical associations of scleritis and glaucoma. Am $\mathcal{F}$ Ophthalmol 1981; 91: 697-705.

3 Bonin P. Cases reported at the Fan Club Meeting, Bonn, November 1984. Case 2. Acute posterior scleritis. Int Ophthalmol 1985; 8: 217-20

4 Cleary PE, Watson PG, McGill JI, Hamilton AM. Visual loss due to posterior segment disease in scleritis. Trans Ophthalmol Soc UK 1975; 95: 297-300.

\title{
Ocular albinism with unilateral sectorial pigmentation in the fundus
}

\author{
Takashi Shiono, Tsutomu Mutoh, Yasushi Chida, Makoto Tamai
}

\author{
Department of \\ Ophthalmology, Tohoku \\ University School of \\ Medicine, 1-1 Seiryo- \\ machi, Aoba-ku, Sendai \\ 980, Japan \\ T Shiono \\ T Mutoh \\ Y Chida \\ M Tamai \\ Correspondence to: \\ T Shiono, MD. \\ Accepted for publication \\ 24 November 1993
}

A diagnosis of ocular albinism ${ }^{12}$ is usually made in individuals with nystagmus, diminished visual acuity, reduced iris and fundus pigment, and foveal hypoplasia. Hypopigmentation of the hair and skin is not found in people with ocular albinism.

We describe a Japanese woman who had ocular albinism with good visual acuity in the left eye and no nystagmus. Her right eye showed low visual acuity. Interestingly, sectorial pigmentation, which reached in part to the macula, was seen only in her left fundus. No pigmentation was noted in the right fundus. To the best of our knowledge, no reports have been written previously about patients with this type of asymmetrical ocular albinism and good visual acuity.

\section{Case report}

A 60-year-old Japanese woman was referred to our clinic on 15 March 1991, for an ophthalmic examination after retinal changes were discovered during a routine examination of her fundus. She had mild diabetes mellitus, no ocular complaints, and no hearing loss. She had been aware of her hypopigmented irides since childhood. Her parents had brown irides. Their marriage was not consanguineous. The patient's three siblings and two daughters had brown irides. No one in her family was reported to have had nystagmus or other eye diseases including hypopigmentation.

Examination showed the patient to have dark brown hair, brown eyebrows, and brown eyelashes. Her skin was not albinotic. No spontaneous nystagmus or strabismus were detected. Her visual acuity was $20 / 40$ in her right eye, with a refractive correction of $-7 \cdot 75 \mathrm{D}$, and $20 / 20$ in her left eye, without correction; however, no stereopsis was found with use of a Randot stereotest. Slit-lamp examination of the anterior segment showed bluish-grey irides with a brown 